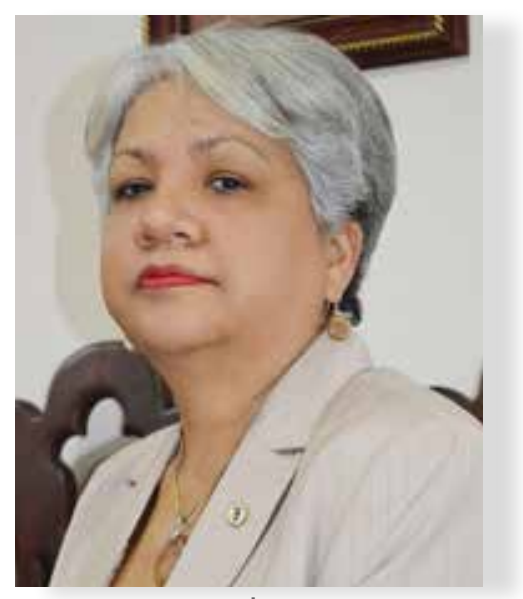

Dra. Sara Güilamo Jiménez

\section{Proyecto Ciudadano. Una estrategia para desarrollar empoderamiento social en el ciclo de los Estudios Generales y el currículo por competencias}

\author{
Proyecto Ciudadano. A strategy to promote civic \\ engagement through liberal arts curricula and competency- \\ based education models
}

Recibido: 07-04-17

Aprobado: 26-09-17

\title{
Resumen
}

En este artículo se describe la importancia de la estrategia de proyecto para el desarrollo del pensamiento crítico y el empoderamiento social, a partir de la experiencia del Consorcio de Educación Cívica de la PUCMM y su programa de Proyecto Ciudadano que desarrolla desde hace más de 15 años con jóvenes estudiantes y líderes comunitarios. Tiene como objetivo conceptualizar la Estrategia de Proyecto, sus características y ventajas para el desarrollo de la competencia Ética y Ciudadanía.

Proyecto Ciudadano se implementa en seis etapas: identificar problemas de políticas públicas en la comunidad; seleccionar democráticamente el más importante; recoger información sobre el problema escogido; planificar la exposición del proyecto; presentarlo ante una audiencia auténtica y llevar a cabo una reflexión sobre el proceso llevado a cabo.

Este artículo presenta la importancia de la estrategia y recoge los testimonios del impacto en los participantes en cuanto al desarrollo de la conciencia ciudadana y compromiso social. Concluye recomendando la Estrategia de Proyecto como una opción metodológica para fortalecer el enfoque de Formación por Competencias y el ciclo de los Estudios Generales

\section{Abstract}

This article describes the importance of project strategy for the development of critical thought and social empowerment as per the experience of PUCMM's Consorcio de Educación Cívica and Proyecto Ciudadano, a citizenship empowement program it has been developing with community leaders over the past 15 years. Proyecto Ciudadano's work revolves around six phases: identifying public policy issues at the community level; democratically selecting the most relevant and important issue; information gathering; planning the presentation of the issue; presenting the issue to the community, and reflecting on the experience. This article describes the strategy and gathers testimonies on the impact of the project in the development of a commitment to the community's wellbeing. It concludes by recommending Project Strategy as a methodological option for the strengthening of university level training in the liberal arts and competency-based models.

\section{Palabras clave}

estrategia de proyecto; proyecto ciudadano; empoderamiento social; formación por competencia; Estudios Generales.

\section{Keywords}

Project Strategy; Proyecto Ciudadano; Social empowerment; Competency-based education; Liberal Arts

*Dra. Sara Güilamo Jiménez: Doctora en Educación por la Universidad de Murcia y tiene Maestría en Educación, Especialidades en Educación Moral y Cívica, Socio pedagogía y Psicología Industrial; Licenciatura en Psicología con amplia experiencia en formación docente. Es directora del Consorcio de Educación Cívica de la PUCMM. Para comunicarse con la autora: saraguilamo@pucmm.edu.do. 
"Un proyecto es un lugar al que queremos ir, pero también es el camino que hay que recorrer para llegar a él”

Jiménez, 2004, en Blanchard \& Muzás, 2016

\section{Introducción}

El Consorcio de Educación Cívica de la PUCMM aglutina un grupo de instituciones y personas interesadas en promover y fortalecer la formación ciudadana con los diferentes actores de la comunidad educativa dominicana, a fin de contribuir a la construcción de una sociedad democrática y participativa que permita el ejercicio de una ciudadanía responsable, a través de la educación cívica. Nació como parte del Proyecto de Iniciativas Democráticas que se desarrolló en los años 80. El Centro Cívico de Los Ángeles de Estados Unidos fue el impulsor y mentor del desarrollo de este programa llamado Proyecto Ciudadano, el cual se comenzó a implementar en varias escuelas dominicanas con su asesoría.

Proyecto Ciudadano contribuye a desarrollar habilidades y destrezas investigativas en el proceso de describir un problema y captar fuentes que den explicación al mismo; desarrolla el pensamiento crítico, porque obliga a analizar los problemas de una manera integral tomando en cuenta los contextos nacionales y locales (sociales y políticos) en que se generan; estimula la comunicación efectiva, la tolerancia y el trabajo en equipo, tanto entre sus compañeros de aula, como entre los demás miembros de la comunidad educativa; fomenta el pensamiento reflexivo ya que promueve el análisis de los problemas en torno a los deberes y derechos adquiridos como ciudadanos; favorece que los alumnos puedan ejercer sus responsabilidades y compromiso como ciudadanos en un estado de derecho; y, promueve el desarrollo de la autoestima, toda vez que hace del estudiante y su proceso de aprendizaje algo vivido y con sentido para la vida personal y profesional.

La experiencia que recoge este estudio comienza con la descripción del proceso dividido en varias etapas de investigación y análisis en torno a un problema de la comunidad o del centro educativo. Luego, presentamos algunos de los resultados de evaluaciones de impacto que se han realizado con los participantes; para terminar, hacemos la vinculación de los aportes que hace Proyecto Ciudadano al proceso de aprendizaje y al currículo por competencias que impulsamos en el sistema educativo dominicano en estos momentos.

\section{Objetivo}

El objetivo que se propone este trabajo es analizar la Estrategia de Proyecto Ciudadano y las ventajas de su uso en los diferentes niveles educativos a partir de la experiencia del Consorcio de Educación Cívica. Se coloca dicha estrategia como una de las opciones metodológicas para el desarrollo de competencias ciudadanas en el nivel superior, de manera específica en el Ciclo de Estudios Generales debido a su eficacia para desarrollar conocimientos, habilidades y actitudes.

\section{Fundamentación teórica}

Es difícil ubicar la estrategia de proyecto en una teoría educativa específica, ya que es una estrategia que se apoya en varias teorías a la vez, tanto en las tradicionales, como las últimas que se han planteado como alternativas. Los enfoques sistémicos (ecológicos/ complejos) son los que más se adecuan para conceptualizarla. Un sistema no puede ser explicado por el simple agregado de sus elementos, sino "un conjunto de elementos que se encuentran en interacción, de forma integral, que produce nuevas cualidades con características diferentes, cuyo resultado es superior al de los componentes que lo forman y provocan un salto de calidad" (Ayma, citado por Sada, Cánovas \& Robles, 2010, p. 416). La teoría de la complejidad de Edgar Morín, utilizada en muchas universidades que han adoptado el enfoque por competencias, es una teoría cónsona con la estrategia de proyecto por sus cualidades integradoras y de articulación con el contexto social. Por su parte, Blanchard y Muzás (2016, p. 47) la consideran más que una estrategia metodológica, por su carácter globalizador que pone en diálogo tres elementos fundamentales para lograr un aprendizaje significativo y vinculado con la vida: los estudiantes, el currículo y el contexto.

La estrategia de proyecto fue ideada originalmente por John Dewey y desarrollada y sistematizada por Kilpatrick en el 1918 como un procedimiento dinámico de organizar la enseñanza mediante actividades con verdadero sentido vital para los estudiantes (Blanchard \& Muzás, 2016). Se originó en la enseñanza agrícola en Estados Unidos (De Miguel, 2006). Tobón (2009) la define como "un plan de trabajo integrado y libremente elegido cuyo objetivo es realizar un conjunto de acciones enmarcadas en la vida real que interesan tanto a los estudiantes como al docente, por lo que despierta el entusiasmo en torno a su ejecución" (p. 129).

En esta estrategia se pueden integrar fases $u$ otras estrategias que incluyen la problematización, indagación y la resolución de problemas; se desarrollan competencias basadas en conocimientos y 
capacidades intelectuales, como las académicas y las del mundo profesional; competencias basadas en el desarrollo de habilidades y destrezas transversales, ya sean intelectuales, de comunicación, interpersonales o de organización y gestión personal; y competencias relacionadas con el desarrollo de actitudes y valores, como son el desarrollo y compromiso personal. (De Miguel, 2006).

El Instituto Tecnológico y de Estudios Superiores de Monterrey (2005) explica los pasos a seguir para llevar a cabo la estrategia de proyecto, teniendo en cuenta que esta requiere de una planificación previa porque demanda mucho tiempo y organización. Lo primero es definir el alcance del proyecto, si es sobre un tema, una asignatura, si involucra varias asignaturas y docentes, si se desarrollará durante todo el semestre o solo un período; en la planificación del proyecto debe resaltarse la autonomía que asumirán los estudiantes y se recomienda ofrecerles gradualmente la guía que necesitarán. El siguiente paso es definir las metas u objetivos a lograr por los estudiantes, que pueden ir desde los aprendizajes de contenidos generales, pasando por los específicos, hasta el "aprendizaje de servicio". Luego, y de acuerdo con los objetivos específicos, se debe definir los resultados esperados en términos de conocimientos y desarrollo de habilidades durante el proceso del proyecto.

Un siguiente paso consiste en definir las preguntas guías, las cuales deben ser provocativas y retadoras, que obliguen a la búsqueda de información y que impliquen desarrollar altos niveles de pensamiento antes de ser contestadas. Estas preguntas deben ser sobre problemáticas reales que interesen a los estudiantes, de donde surgen otras preguntas que serán cada vez más específicas. Esto genera las actividades potenciales para la búsqueda de información que darán respuesta a las preguntas. Es muy importante definir los productos esperados en el desarrollo del proyecto, a sabiendas de que pueden generarse otros durante el proceso, como son: ensayos, instrumentos de recogida de información, cronogramas de trabajo, reportes, guiones, entre otros. Se procede entonces al desarrollo de las actividades de aprendizaje planificadas, las cuales pueden ser organizadas en bloques y por fases, para facilitar su evaluación.

En cuanto al rol docente, es muy importante ofrecer el apoyo instruccional, "con el fin de guiar el aprendizaje de los alumnos, así como facilitar un exitoso desarrollo del producto del proyecto" (El Instituto Tecnológico y de Estudios Superiores de Monterrey, 2005, p. 14). El docente aporta orientación, lectura, demostración y modelos que ayuden a los estudiantes; Este proceso inicial le llaman "andamiaje", y constituye el puente entre las competencias de los alumnos y lo que se requiere para lograr los aprendizajes del proyecto. El último paso se dedica a describir las características que debe poseer el ambiente de aprendizaje para el desarrollo del proyecto; debe cambiarse, preferiblemente, el ambiente de aula a un espacio de oficina o salón de reuniones; garantizar que los grupos de trabajos sean heterogéneos. Es necesario prever la posibilidad de que algunas actividades se desarrollarán fuera del aula o en otra institución. Es muy importante también identificar los recursos con que se cuenta para el proyecto (cámaras, computadoras, libros, entre otros).

Como vemos, el rol del docente consiste fundamentalmente en prepararse para manejar bien la estrategia, motivar y acompañar a los estudiantes durante todo el desarrollo del proyecto. Es importante la identificación de la literatura más pertinente para el tema y la dirección a la búsqueda de fuentes seguras de información, también se debe poner a la disposición recursos tecnológicos, así como se debe elaborar los instrumentos de evaluación. Por su parte, como hemos planteado, los estudiantes tienen que asumir un rol protagónico, pero esto no significa que le dejemos solos. Ellos deben aprender a organizarse y actuar con mucha disciplina para cumplir con lo planeado en el proyecto. La dinámica que se da en los grupos responde al trabajo colaborativo, el cual

es un proceso en el que las partes se comprometen a aprender algo juntas. Lo que debe ser aprendido sólo puede conseguirse si el trabajo del grupo es realizado en colaboración. Es el grupo el que decide cómo realizar la tarea, qué procedimientos adoptar, cómo dividir el trabajo o tareas a realizar. La comunicación y a negociación son claves en el proceso. (Gros, 2000, en Maldonado Pérez, 2007, p. 269)

De acuerdo a estos pasos descritos, la estrategia de proyecto permite desarrollar un aprendizaje anti dogmático, integral y autónomo; los estudiantes participan con una motivación intrínseca, que a su vez previene y resuelve conflictos interpersonales por la confianza que desarrollan en sus propias habilidades y talentos; promueve la asimilación de conceptos, valores y formas de pensamiento; establece un clima de apoyo entre los estudiantes; provee medios para transferir la responsabilidad del aprendizaje de los docentes a los estudiantes en forma completa o parcial; permite a los estudiantes tratar nuevas habilidades y modelar conductas complejas; los invita a explicar o defender su posición ante los demás en sus proyectos grupales, para que su aprendizaje sea personal y puedan valorizarlo, y sirve como un medio para envolver a los estudiantes que usualmente no participan. Una ventaja adicional de la estrategia es su compatibilidad con los diferentes estilos de aprendizaje, existen múltiples 
maneras para los estudiantes participar y demostrar su conocimiento en actividades como: lectura y revisión de documentos, participación en discusiones, preparar datos estadísticos, ejercitar su liderazgo, entre otras. (Instituto Tecnológico y de Estudios Superiores de Monterrey, 2005)

En relación a los recursos materiales y didácticos que se requieren para desarrollar la estrategia de proyecto, presenta la ventaja de que pueden utilizarse todos los que se tengan a la disposición, además de que puede integrar los que lleven los estudiantes desde sus casas, dependiendo del tema en cuestión. Nos referimos a libros y artículos de consulta disponibles en la biblioteca, laboratorios, manipulativos, recursos audiovisuales y tecnológicos, entre otros.

La evaluación de los proyectos también debe ser una corresponsabilidad de los estudiantes participantes y debe hacerse de manera procesual. Se debe priorizar las técnicas que lleven a la meta cognición, o sea, aquellas en las que los estudiantes puedan hacer consciencia de sus propios aprendizajes. Es importante que se sistematice la experiencia completa, donde se preparen informes, diarios reflexivos, presentaciones orales de resultados parciales, recopilación de documentos y evidencias importantes.

Existen diferentes tipos de proyectos o proyectos con diferentes calificativos como son: participativos, colaborativos, científicos, tecnológicos y ciudadanos. Son estos últimos los que pretendemos desarrollar aquí.

En los proyectos ciudadanos, los estudiantes actúan como ciudadanos inquietos y críticos, que solidariamente consideran los problemas que los afectan, se informan, proponen soluciones y, de ser posible, las ponen en práctica o las difunden, así sea a pequeña escala. Como ejemplos de este tipo de proyectos podemos mencionar el estudio de hábitos nutricionales de compañeros del plantel, la investigación sobre posibilidades recreativas para niños en la comunidad, o la detección de fuentes de contaminación en la periferia de la escuela (Hurd,1982; Aikenhead, 1996 \& Fensham, 1997, en Perrenoud, 2006, pp. 16 y 17).

El proyecto ciudadano contribuye a formar ciudadanos y ciudadanas conscientes, responsables y críticos de la realidad, para que sean capaces de promover cambios en su entorno desde temprana edad. Es una forma de hacer ciudadanía activa, hacerse conscientes de sus deberes y derechos y desarrollar un comportamiento autónomo. Les permite conocer a cabalidad las principales políticas públicas existentes, comenzando con el marco jurídico general que es la Constitución de la República y los Derechos Humanos.
Asimismo, ponen en práctica los procedimientos para solicitar la revisión de algunas normativas o proponer la elaboración de otras al Congreso Nacional.

Esta es una estrategia basada en la interdisciplinariedad y el fomento de la reflexión, y tendente a formular propuestas para la solución de problemas de la comunidad, con la participación de los actores involucrados para fomentar una ciudadanía social responsable. Pretende promover la vinculación de las diferentes instancias educativas con la comunidad. Se desarrolla generalmente con el apoyo de los docentes, pero involucrando a los estudiantes desde la primera fase del proceso. Esta estrategia ayuda a asumir sus responsabilidades y a demandar el cumplimiento y respeto de sus derechos, con el fin de incidir en políticas públicas a través de la identificación de problemas en la comunidad y dar un aporte con su propuesta de solución.

\section{Contextualización}

El Consorcio de Educación Cívica comenzó la aplicación de Proyecto Ciudadano junto al Centro Cívico de los Ángeles, Estados Unidos, quienes fueron invitados a conocer el proyecto para ver la factibilidad de aplicarlo en República Dominicana. Fue así como algunos de sus miembros recibieron capacitación y sirvieron de multiplicadores para los demás.

La estrategia implementada dispone de un manual del estudiante y una guía para el docente o facilitador, los cuales fueron traducidos y adaptados con la asesoría de ellos. Luego, se iniciaron las capacitaciones a docentes del Área de Ciencias Sociales del Ministerio de Educación; durante los talleres se fueron validando los manuales y replicando el proceso con sus estudiantes.

El objetivo principal de un proyecto ciudadano es participar en el desarrollo de una política pública que pueda resolver problemas comunitarios partiendo de una investigación profunda de los mismos. Ayuda a los estudiantes a ejercer una doble dimensión de la ciudadanía: asumir responsabilidades y demandar el cumplimiento y respeto de sus derechos. Es un proceso reflexivo y activo. En principio se desarrolló con estudiantes del Nivel Secundario del sector público. Hoy en día ya tenemos varios grupos de estudiantes del Nivel Primario (2do. ciclo) que han desarrollado proyectos ciudadanos exitosamente.

De manera obligada la implementación de Proyecto Ciudadano va articulada a los Derechos Humanos Fundamentales, particularmente a la Constitución de la República Dominicana. Todas las actividades que se lleven a cabo tienen que estar dentro del marco de respeto a los derechos y deberes establecido en ese marco jurídico. Esta es la razón por la que la estrategia 
de proyecto tal y como se aplica en el Consorcio de Educación Cívica es recomendada a los ministerios de Educación y el de Educación Superior Ciencia y Tecnología ya que contribuye a impulsar el pensamiento profundo y la formación por competencias. Sanz de Acedo $(2010$, p. 91) explica que: "las competencias de toma de decisiones y de solución de problemas son las más complejas del pensamiento, son las denominadas competencias superiores; por ello, han de ser ejercitadas y estimuladas en todos los planes y niveles de estudio de la Educación Superior". A través de ella se desarrolla el aprendizaje para toda la vida. Al respecto, Blanchard y Muzás enfatizan que “...es fundamental que los contenidos que ofrecemos para ser aprendidos, le sirvan al niño, y al adolescente para la vida real, para dar respuesta a lo que le sucede, en las situaciones ordinarias, en el aquí y en el ahora, y no tenga que esperar a que aquello que ha aprendido le sirva, como nos decían antes: "Tu apréndelo, que ya verás cómo algún día te sirve”. (2016, p. 67).

\section{Metodología}

Para implementar un proyecto ciudadano se inicia con un taller para la capacitación de los docentes - líderes comunitarios que servirán de apoyo replicando lo aprendido con los grupos de jóvenes. Primero se explica en qué consiste la metodología y la importancia que tiene para poner en práctica los valores ciudadanos. Se conceptualiza lo que es una política pública y se modela cada uno de los pasos que se desarrollarán con los estudiantes. Este primer taller culmina con la asignación de la investigación sobre el problema seleccionado por los participantes. En el segundo taller los estudiantes hacen el análisis de sus datos y preparan la presentación oral utilizando recursos visuales y la carpeta. De este proceso debe generarse la motivación y el compromiso de los participantes de ser multiplicadores en los grupos de clase o de su comunidad. Para esto desarrollan un cronograma de aproximadamente seis meses en los espacios reservados en su calendario escolar para los contenidos de Ética y Ciudadanía. Finalmente, llevan a cabo el proyecto en su contexto y con el acompañamiento nuestro.

\section{Identificar problemas de Políticas Públicas}

Con la ayuda del manual se ofrece literatura pertinente para discutir el concepto de Política Pública y construir su propia definición y múltiples ejemplos. Se produce, entonces, una lluvia de ideas sobre problemas existentes en el centro o en su comunidad para los cuales es necesario generar una propuesta de política pública. Consultan con sus padres, maestros y relacionados, además de compartir con sus compañeros lo que saben sobre esos problemas.

El grupo genera una lista de problemas, la cual debe evaluar a partir de la lectura de distintas fuentes de información para analizar y sintetizar con base en lo que conviene a la mayoría. Para los fines, se le proporciona un formulario para registrar cada uno de los problemas que propondrá para su análisis.

Los problemas más frecuentes planteados por los jóvenes son: insalubridad, deficiencia de servicios básicos, distribución y consumo de drogas (legales e ilegales), embarazo en adolescentes, falta de condiciones para la docencia en los centros educativos (mobiliario, equipos, higiene), aborto, maltrato infantil, problemas de convivencia en los grupos, contaminación del medio ambiente en todas sus manifestaciones, entre otros.

\section{Seleccionar un problema}

Una vez se recibe esta lista de problemas por parte de los alumnos, toca reflexionar con ellos para seleccionar el más importante para la comunidad. El proceso de selección debe ser democrático y dirigido por ellos. Se comparte el concepto sistema democrático y se analizan otros como el de justicia, autoridad, respeto por la diversidad. Con las deliberaciones sobre el impacto de los problemas propuestos se trata de reducir la cantidad. A veces toman la decisión de manera unánime, sin que haya que someterla a votación. De lo contrario, se organizan las votaciones hasta quedar con uno.

El proceso de toma de decisiones es uno de los más importantes por ser una competencia cognitiva de orden superior, sobre todo cuando es en grupo, donde se deben tomar en cuenta factores personales de los participantes, las complejidades propias de la tarea y el entorno en que se presenta el problema. Para ello se tienen que agotar varias fases: asumir el compromiso para hacer el planteamiento de problema, hacer una representación mental para planificarlo, especificar los objetivos que persigue, generar las diferentes opciones que existen para resolverlo, evaluar cada una de ellas (sus pros y contras) para finalmente elegir la mejor de esas opciones. (Sanz de Acedo, 2010)

Elegido el problema, el profesor debe tener en cuenta que este no constituya peligro para los alumnos cuando vayan a recoger información, por ejemplo, temas como el de las pandillas delictivas. "...cuando pertenecen a un grupo, las personas están dispuestas a aceptar mayores riesgos que los que por prudencia o temor toman cuando acometen una tarea individualmente..." (Sanz de Acedo, 2010, p. 94) 


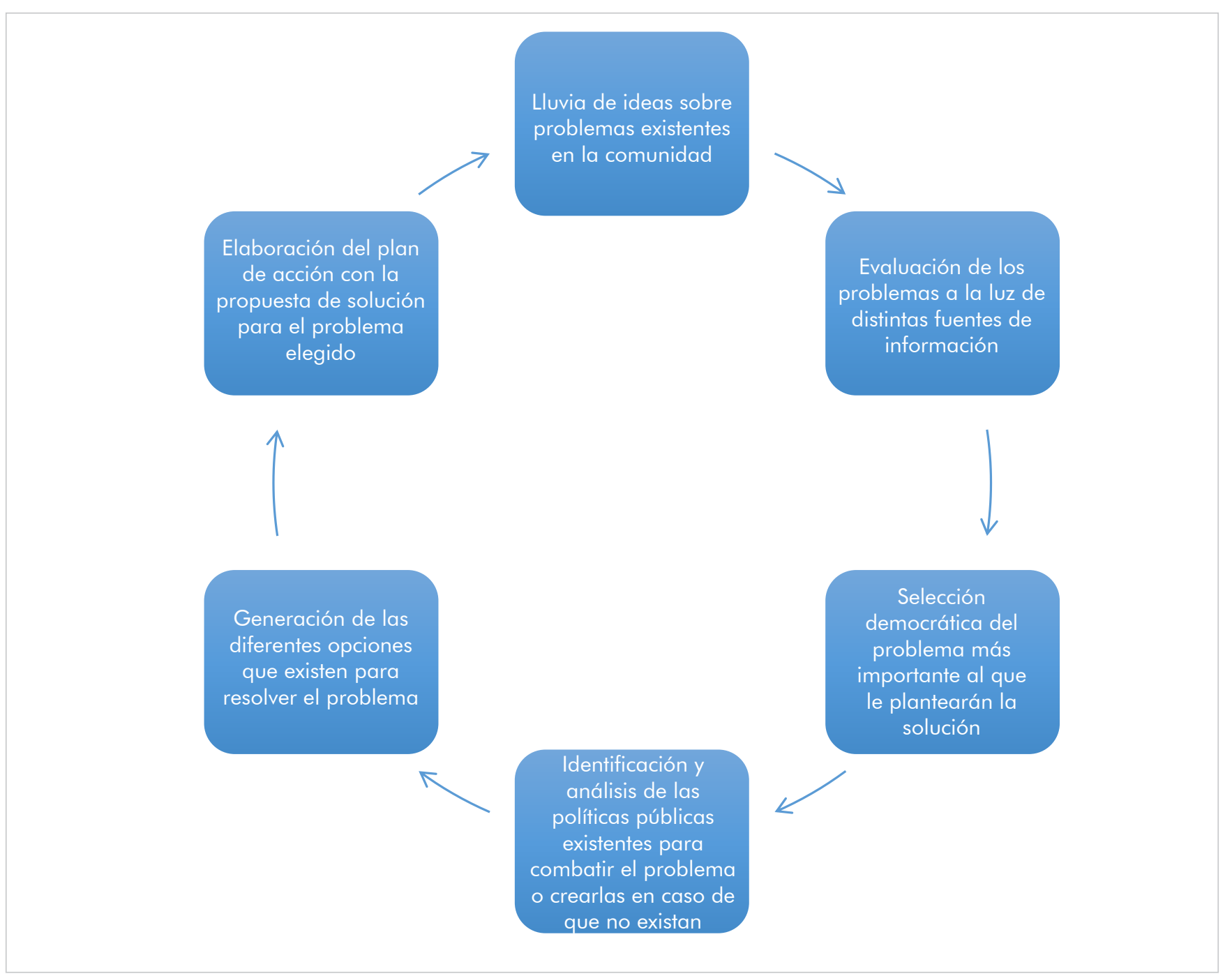

Figura 1: Fases del proyecto para la elección del problema y presentación de la propuesta.

\section{Recolección de la información sobre el problema a estudiar}

En esta etapa se recogen las informaciones sobre el tema, las cuales son de dos tipos: documental y de campo. El docente o líder ayuda en el proceso completo. Para la ubicación y selección de las fuentes a consultar, por ejemplo, artículos científicos que contribuyan a conceptualizar el problema y a ubicar sus antecedentes. También, información sobre las leyes y otras políticas públicas existentes en torno al problema de estudio.

La recolección de información de campo generalmente se hace utilizando cuestionarios, entrevistas a informantes claves de la comunidad, o a funcionarios públicos (porque deben averiguar las instancias del gobierno responsables de resolver el problema objeto de estudio). El docente debe garantizar que sean acompañados de adultos para visitas a instituciones, pero si se implementa en el nivel superior, se contaría con la independencia natural de los jóvenes universitarios. El docente asume su rol participando en la revisión de los instrumentos y guías. Es un momento propicio para la interdisciplinariedad al involucrar a docentes de otras áreas. Por ejemplo, el área de lenguas puede participar ayudando en la redacción de cartas de solicitud de entrevistas y demás documentos; el área de matemáticas, con las tabulaciones de las respuestas a las preguntas del cuestionario, elaboración de tablas y figuras.

Este proceso termina cuando se recopilan las informaciones, se clasifican y analizan hasta producir un plan de acción que se presentará como propuesta de solución. El análisis debe hacerse en torno a qué tan importante es ese problema para la comunidad y por qué; cuáles son las instancias estatales responsables de resolverlos; si existen políticas públicas al respecto que deban movilizarse, o proponer la creación de políticas nuevas; entre otras. 

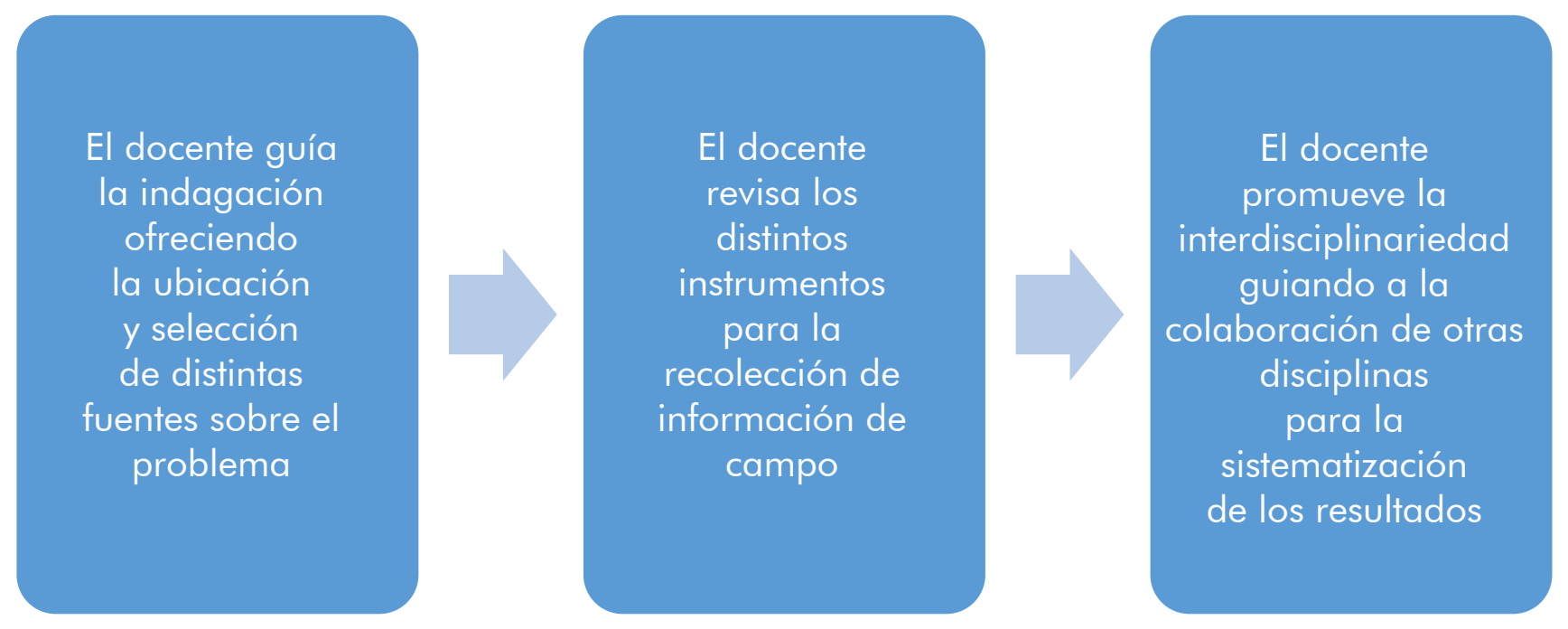

Figura 2: Rol docente para la recolección de la información del problema.

\section{Presentación del proyecto}

Para la presentación del proyecto el curso se divide en cuatro equipos: explicación del problema, análisis de las políticas existentes, política propuesta y plan de acción. Deben prepararse juntos porque la responsabilidad de cada equipo está relacionada con la de los otros. Hay que garantizar que participen todos los estudiantes. No está permitido seleccionar para la presentación sólo los que tienen más habilidades verbales.

\section{Equipo 1 Explicación del problema}

En no más de dos páginas describen y explican la gravedad del problema, donde está localizado y justifican por qué necesita solución. Anuncian los responsables de resolverlo y las leyes e instancias relacionadas, así como las diferentes posturas e intereses que existen y que pudieran ser obstáculos para resolverlo.

\section{Equipo 2 Análisis de las políticas existentes}

Es responsable de evaluar y explicar claramente las políticas existentes, sus fortalezas y debilidades, así como las alternativas que se han ofrecido para resolver el problema. Debe incluir un resumen escrito de las políticas, además de anexar las leyes relacionadas. Se plantean preguntas como: ¿Por qué existe el problema?; ¿Desde cuándo?; ¿Cuáles son las instituciones responsables de solucionarlo?; ¿Qué han hecho hasta el momento?; ¿Por qué no fueron efectivas esas políticas?, entre otras.
Equipo 3 Política propuesta o sugerida

Su responsabilidad es explicar la política que propone el grupo para solucionar el problema.

Puede ser que hayan desarrollado su propia política, o que hayan decidido apoyar una de las existentes con alguna modificación. En ocasiones apoyan una y demandan su correcta aplicación si es lo que procede, ya que es muy frecuente en nuestro país, contamos con leyes y procedimientos bien elaborados, sin embargo, no se aplican.

\section{Equipo 4 Plan de acción}

Este equipo explica el plan de acción. Incluye los pasos que deben dar para que el gobierno acepte y ponga en práctica su política propuesta. Debe ser por escrito y con una estrategia que permita lograr el apoyo de personas y organizaciones de la comunidad para resolver el problema. En este proceso ya han identificado también las personas e instancias del gobierno que estarían dispuestas a apoyar su política sugerida. Identifican las posiciones y grupos que podrían oponerse, y explican qué harían con esos obstáculos.

\section{Exhibición}

Previo al inicio de la presentación, el salón ha sido preparado para el evento y se reserva un espacio para los evaluadores, uno para el público y otro donde se hará la presentación con un recurso visual y una mesa para el portafolio o carpeta. 
El recurso visual consiste en un cartel de cuatro láminas grandes de cartón, y cada una es utilizada por un equipo para exhibir sus producciones. Pueden colocarse escritos, documentos, fotos, figuras y tablas estadísticas, según consideren.

Igualmente, se procede a preparar la carpeta, dividida en cuatro secciones correspondientes a los cuatro equipos. El grupo decide qué materiales incluir en el cartel y cuáles en la carpeta. Ese día también exhiben libros y otros materiales que utilizaron para su investigación.

\section{Exposición al público}

La preparación del evento incluye la invitación de una serie de expertos en Proyecto Ciudadano que fungen como evaluadores del proceso. Para los fines utilizan unas rúbricas para cada equipo que presenta.

Por otra parte, se invitan autoridades que estén relacionadas con el problema de investigación; así como autoridades educativas, las cuales siempre estarán valorando los aspectos formativos del proceso.

Su profesor o líder inicia la actividad explicando qué es Proyecto Ciudadano. A seguidas, los estudiantes dirigen toda su presentación. Se espera que las participaciones de los evaluadores, autoridades y público sean de estímulo y motivación para los estudiantes. En ocasiones, las autoridades han hecho promesas en el momento para resolver algún problema presentado.

Finalmente, hacen una reflexión sobre la experiencia de desarrollar el proyecto a partir de las siguientes preguntas, las cuales forman parte del contenido del Manual del Estudiante:

1. ¿Qué aprendí personalmente sobre las políticas públicas al trabajar con mis compañeros/as?

2. ¿Qué aprendimos como grupo sobre las políticas públicas al desarrollar el proyecto?

3. ¿Qué habilidades obtuve o mejoré a partir de este proyecto?

4. ¿Qué habilidades obtuvimos o mejoramos a partir de este proyecto?

5. ¿Cuáles son las ventajas de trabajar en grupo?

6. ¿Cuáles son las desventajas de trabajar en grupo?

7. ¿Qué hice bien?

8. ¿Qué hicimos bien?

9. ¿Cómo puedo mejorar mis habilidades para resolver problemas?

10. ¿Cómo podemos mejorar nuestras debilidades?
11. ¿Qué haríamos de otra manera si tuviéramos que desarrollar una política pública sobre otro tema?

Estas preguntas son respondidas espontáneamente por los miembros del grupo, en público, al finalizar su presentación.

\section{Testimonios del impacto de proyecto ciudadano}

Los resultados de esta experiencia capacitando y acompañando docentes y líderes juveniles para desarrollar Proyecto Ciudadano en sus grupos podemos verlos a corto y largo plazo; a sabiendas de que quizás los aprendizajes más valiosos son intangibles, porque se trata de instalar valores y actitudes. Aproximadamente quince años recorriendo centros educativos, comunidades y grupos diversos, nos ha permitido recoger la percepción inmediata de los participantes (utilizando rúbricas de evaluación); así como al contactar algunos participantes, después de adultos y profesionales, que nos testimonian haber elegido una carrera del Área de Ciencias Sociales motivados por su participación en dicho proceso.

Los testimonios que ofrecen los participantes (estudiantes y docentes) expresan su experiencia de aprendizaje:

con el trabajo de Proyecto Ciudadano nos han enseñado que nosotros podemos cooperar con nuestra comunidad...pude darme cuenta que yo podía hacer encuestas, o sea, hacerle preguntas a personas que no son mis compañeros o mis familiares...nunca habíamos visitado un destacamento de la Policía y para hacer el trabajo de Proyecto Ciudadano lo visitamos por primera vez - expresan los estudiantes. (Consorcio Educación Cívica, 2007, p. 39).

Reportan además vivencias tan interesantes como: "aguantamos empujones de los periodistas, pero logramos entrevistar al Secretario de Salud Pública para nuestro trabajo de Proyecto Ciudadano", o: "fue interesante para nosotros la visita a la oficina de Educación y Medio Ambiente de la Circunscripción II del Ayuntamiento del D.N. pues no nos imaginábamos que existía y lo bien organizada que está". Proyecto Ciudadano también aporta a la integración de los padres en el proceso de aprendizaje de sus hijos: "para mí fue una linda experiencia acompañar a mis hijos y sus compañeros al destacamento de Gualey y a la Fiscalía Barrial a buscar informaciones para el trabajo..." (Consorcio Educación Cívica, 2007, pp. 39 y 40) 
A continuación, presentaremos como ejemplo algunos testimonios de docentes y estudiantes refiriéndose a su experiencia con el programa de Proyecto Ciudadano.

Los docentes reportan cómo desarrollaron el proyecto, el protagonismo de los estudiantes para llevar y seleccionar la problemática a estudiar, como "el ruido, la delincuencia, las drogas". Ellos explican cómo formaron comisiones de los estudiantes y se trasladaron con ellos a los diferentes lugares a buscar información. "Por ejemplo, en cuestión de los ruidos buscamos a las autoridades competentes, porque algunos estudiantes tenían padres involucrados, ya ahí hay una problemática, cada representante de juntas de vecinos, algunos hasta funcionarios, y se logró reparar un poquito los daños de la problemática".

Continúan explicando los docentes en relación a la participación de los estudiantes en el programa:

algo importante que quisiera destacar es cómo se fueron fomentando las relaciones entre organizaciones del estado, los alumnos y las juntas de vecinos, siendo uno de los indicadores del empoderamiento comunitario"; “... entonces hacíamos una especie de diagnóstico y a medida que hacíamos el diagnóstico, los estudiantes iban detectando los problemas que se presentaban, entonces, con base en eso elaboramos algunos proyectos donde se planteaban los propósitos, lo que se quería lograr y eso, por ejemplo, desarrollamos uno sobre la delincuencia, cómo eso afecta al sector y a la comunidad. Estuvimos trabajando también el problema del medio ambiente. Es decir, fueron detectando problemas que se van dando y que ellos van tomando conciencia sobre esa problemática, porque para uno lo más importante es eso de que el ciudadano y la ciudadana puedan hacer conciencia de cuál es la responsabilidad con el entorno en donde viven, donde se desarrollan. (Dotel et al., 2009, p. 34).

Los estudiantes, por su parte, también explicaron la forma en que se involucraron junto con su centro educativo en actividades, independientemente del programa, en beneficio a la comunidad: "nosotros salíamos en una jornada por todo Buenos Aires y les decíamos que tienen que untarle cloro y tapar los tanques, que tienen que tener cuidado". (Dotel et al., 2009 , p. 34). El equipo referido recalca dos palabras muy importantes que caracterizan el programa y que se reflejan en su investigación, que son el hecho de crear conciencia y responsabilidad, siendo estos los propósitos del programa.
Aporte del proyecto ciudadano al desarrollo integral de los estudiantes

En todos los niveles del currículo en estos momentos se están haciendo adecuaciones para asumir el Enfoque por Competencias. Hemos visto cómo Proyecto Ciudadano y su metodología puede ser una excelente estrategia pedagógica para impulsar este nuevo enfoque.

Por la naturaleza de este contexto, resaltamos su aporte para el nivel universitario, y muy específicamente en nuestra Pontificia Universidad Católica Madre y Maestra (PUCMM), que en su Modelo Educativo plantea educar profesionales que amen el conocimiento como instrumento de libertad y de desarrollo personal y social, que posean un sentido ético y una conciencia crítica de la realidad, que propicien acciones a favor de la dignidad humana, que busquen la paz y que promuevan la justicia social y la protección de la naturaleza. En ese orden, en los fundamentos antropológicos que caracterizan el Modelo Educativo, se contemplan valores como: la libertad y autonomía responsable de la persona, la búsqueda de la verdad y la justicia social, la responsabilidad, el bien común, la dialogicidad, el amor y el respeto mutuo. (PUCMM, 2011; PUCMM, 2016).

La PUCMM ha decidido llevar a cabo el Ciclo de los Estudios Generales, los cuales se proponen incidir en las personas para el cambio individual y social; con posturas críticas ante las diversas ideologías e intereses, que faciliten la deliberación pública para la toma de decisiones, conscientes del respeto a las normas de convivencia y promotores de la participación inclusiva; que generen conciencia personal para que el ejercicio ciudadano y profesional se oriente hacia la equidad social y el mejoramiento de la calidad de vida de las personas; que desarrollen las destrezas fundamentales del pensamiento, que permitan la búsqueda y aplicación de soluciones creativas en situaciones complejas. (PUCMM, 2016). Todo esto es factible conseguirlo con la implementación de la estrategia de proyectos ciudadanos.

En la tabla que presentamos a continuación, se presentan las competencias que se plantean desarrollar en el Ciclo de Estudios Generales, clasificadas en tres grupos: instrumentales, interpersonales y sistémicas. Si analizamos las diferentes etapas, desde la planificación, pasando por la investigación y desarrollo del proyecto, hasta la preparación de la carpeta y presentación al público; Proyecto Ciudadano contribuye al desarrollo de todas estas competencias.

En cuanto al currículo de los niveles primario y secundario se plantea la Competencia Ética y 
Tabla 1: Competencias del Ciclo de Estudios Generales

\begin{tabular}{|c|c|c|}
\hline \multirow{4}{*}{ Instrumentales } & Cognitivas & $\begin{array}{l}\text { Pensamiento analítico, sistémico, crítico, reflexivo, } \\
\text { lógico, analógico, colegiado, creativo y deliberativo. }\end{array}$ \\
\hline & Metodológicas & $\begin{array}{l}\text { Resolución de problemas } \\
\text { Toma de decisiones } \\
\text { Orientación al aprendizaje } \\
\text { Planificación }\end{array}$ \\
\hline & Tecnológicas & Manejo de las TIC \\
\hline & Lingüísticas & Comunicación oral y escrita \\
\hline \multirow{2}{*}{ Interpersonales } & Individuales & $\begin{array}{l}\text { Automotivación } \\
\text { Diversidad e interculturalidad } \\
\text { Resistencia y adaptación al entorno } \\
\text { Sentido de trascendencia } \\
\text { Sentido ético } \\
\text { Sensibilidad estética }\end{array}$ \\
\hline & Sociales & $\begin{array}{l}\text { Comunicación interpersonal } \\
\text { Trabajo en equipo } \\
\text { Tratamiento de conflictos y negociación } \\
\text { Responsabilidad social y compromiso ciudadano } \\
\text { Compromiso con la preservación del medio ambiente }\end{array}$ \\
\hline \multirow{3}{*}{ Sistémicas } & Organización & Gestión por objetivos y proyectos \\
\hline & Emprendimiento & Creatividad \\
\hline & \multicolumn{2}{|l|}{ Investigación } \\
\hline
\end{tabular}

Fuente: PUCMM Estudios Generales

Ciudadana como fundamental, además de incluir parte de estos contenidos en el Área de Ciencias Sociales. Explica que la

construcción de soluciones colectivas a los problemas sociales pasa por la construcción de una cultura democrática, que a su vez está basada en unos valores éticos y ciudadanos. La búsqueda de salidas individuales ausentes de solidaridad es una evidencia de las limitaciones que impone la cultura autoritaria para la construcción de soluciones colectivas. Incluso las prácticas políticas tradicionales han reforzado este patrón en variadas formas, tales como el personalismo, el clientelismo y la corrupción administrativa. Esto resulta contraproducente para la vivencia de unos valores democráticos, aunque explicable dada la fuerte herencia de los largos periodos dictatoriales que ha vivido la sociedad dominicana desde su formación...El desarrollo de la Competencia Ética y Ciudadana supone un esfuerzo mayor en los tiempos actuales. La escuela tiene el reto de constituirse en un espacio crítico de esa cultura autoritaria que se perpetúa en las relaciones sociales y de la que la misma escuela participa. Las instituciones educativas deben convertirse en un espacio de construcción de nuevas relaciones sociales, que puedan generalizarse a otros ámbitos de la sociedad. (MINERD, 2013, p. 1)

La evaluación de competencia supone unos indicadores de logros donde se evidencie una práctica de valores. Encontramos criterios como: mostrar responsabilidad y confianza en los demás al trabajar en equipo; criticidad en la interpretación de mensajes que circulan en su ambiente social; pertinencia en los argumentos relativos a valores éticos y ciudadanos; sensibilidad y solidaridad ante el dolor y las necesidades ajenas; respeto por los demás; valoración de las diferencias culturales como fuente de enriquecimiento; criticidad e 
indignación ante prácticas antidemocráticas; frecuencia en la ejercitación de deberes y derechos ciudadanos, entre otros. (MINERD, 2016)

\section{Conclusión}

Como vemos, Proyecto Ciudadano utiliza estrategias cooperativas. Enfoca los aprendizajes de los aspectos gubernamentales en el plano estatal y local; parte de problemáticas reales de la comunidad nacional; provee nuevos modelos de evaluación estudiantil. Los estudiantes aprenden a vigilar, influir e incidir en políticas públicas.

Para los docentes universitarios es una excelente oportunidad de conformar equipos de diferentes disciplinas que integren contenidos, habilidades y actitudes que de manera particular no pueden ser desarrollados con asignaturas independientes. Ya hemos visto que el Enfoque por Competencias debe partir de la integralidad de la educación para mostrar la complejidad del ser humano en particular, y de los procesos sociales en general.

Esperamos que el conocimiento y la aplicación de la estrategia de proyecto sea uno de los ejes de formación docente para que estas fortalezas que hemos presentado tengan mayor impacto en el sistema educativo dominicano.

Y más que una estrategia metodológica, como una metodología globalizadora, que pone en diálogo tres elementos fundamentales para lograr un aprendizaje significativo y vinculado con la vida: por un lado, los intereses de los estudiantes, por otro el currículo establecido $\mathrm{y}$, junto a estos, las necesidades y realidad del contexto en el que se desarrolla la acción educativa". (Blanchard \& Muzás, 2016, p. 47)

Curricularmente hay un reconocimiento de ella, sin embargo, requiere que los docentes sean acompañados en el proceso de implementación y desarrollo de la misma. De esta manera se contribuye a formar ciudadanos/profesionales íntegros y comprometidos con fomentar una cultura democrática en el país.

\section{Referencias}

Blanchard, M. \& Muzás, M.D. (2016). Los Proyectos de Aprendizaje. Un marco metodológico clave para la innovación. Madrid, España: Narcea, S.A.
Consorcio Educación Cívica (2007). 10 años de formación ciudadana en República Dominicana. Documento interno.

De Miguel Díaz, M. (Coord.) (2006). Metodologías de Enseñanza y Aprendizaje para el Desarrollo de Competencias. Orientaciones para el profesorado universitario ante el espacio europeo de educación superior. Madrid: Alianza Editorial.

Dotel, O. (2009). Consorcio de Educación Cívica, Pontificia Universidad Católica Madre y Maestra (PUCMM): 12 años de lecciones aprendidas. República Dominicana: Editora Buho.

Instituto Tecnológico y de Estudios Superiores de Monterrey (2005). Dirección de Investigación y Desarrollo Educativo. Las estrategias y técnicas didácticas en el rediseño. El método de proyectos como técnica didáctica. México: ITESM

Maldonado Pérez, M. (2007). El trabajo colaborativo en el aula. Revista de Educación. Laurus. vol. 13, núm.23, 2007, pp. 263-278. Universidad Pedagógica Experimental Libertador Caracas, Venezuela

MINERD (2013). Dpto. de Curriculum. Competencia ética y ciudadana.

Perrenoud, P. (2006). Aprender en la escuela a través de proyectos: ¿Por qué?, ¿Cómo?. Secretaria de Educación Pública. Primer Taller de Actualización sobre los Programas de Estudio. México, D.F.

PUCMM (2016). Centro de Desarrollo Académico. Núcleo del Plan de Estudios Ciclo de Estudios Generales.

PUCMM (2011). Modelo Educativo. Santiago de los Caballeros, Republica Dominicana: Editora Teófilo.

Sada, E., Cánovas, M., Robles, J. (2010). Retos Internacionales ante la Interculturalidad. España: Publicaciones de la Universidad de Almería

Sanz de Acedo Lizarraga, M.L. (2010). Competencias cognitivas en Educación Superior. España: Narcea, S.A. de Ediciones.

Tobón, S. (2009). Formación Basada en Competencias. Pensamiento Complejo, Diseño Curricular y Didáctica. 2da. Edición. Bogotá: ECOE Ediciones. 